\title{
Th17 cytokine deficiency in patients with Aspergillus skull base osteomyelitis
}

\author{
Corine E Delsing ${ }^{\dagger}$, Katharina L Becker ${ }^{\dagger}$, Anna Simon, Bart Jan Kullberg, Chantal P Bleeker-Rovers, \\ Frank $L$ van de Veerdonk and Mihai G Netea*
}

\begin{abstract}
Background: Fungal skull base osteomyelitis $(\mathrm{SBO})$ is a severe complication of otitis externa or sinonasal infection, and is mainly caused by Aspergillus species. Here we investigate innate and adaptive immune responses in patients with Aspergillus SBO to identify defects in the immune response that could explain the susceptibility to this devastating disease.
\end{abstract}

Methods: Peripheral blood mononuclear cells isolated from six patients with Aspergillus SBO and healthy volunteers were stimulated with various microbial stimuli, among which also the fungal pathogens Candida albicans and Aspergillus fumigatus. The proinflammatory cytokines IL-6, TNFa and IL-1 $\beta$, and the T-helper cell-derived cytokines IFNY, IL-17 and IL-22 were measured in cell culture supernatants by ELISA.

Results: Proinflammatory cytokine responses did not differ between SBO patients and healthy volunteers. The Candida- and Aspergillus-specific Th17 response (production of IL-17 and IL-22) was significantly decreased in the SBO patients compared to healthy individuals, while Th1 cytokine response (IFNy production) did not differ between the two groups.

Conclusions: We show that patients with Aspergillus skull base osteomyelitis infection have specific defects in Th17 responses. Since IL-17 and IL-22 are important for stimulating antifungal host defense, we hypothesize that strategies that have the ability to improve IL-17 and IL-22 production may be useful as adjuvant immunotherapy in patients with Aspergillus SBO.

Keywords: Aspergillus, Skull base osteomyelitis, Interleukin-17, Interleukin-22, Th17 response, Antifungal host defense

\section{Background}

Skull base osteomyelitis (SBO) is a rare but lifethreatening infection, which originates either from the external ear canal or the paranasal sinus. Infiltrative growth from the external acoustic duct into the temporal bone is also termed malignant or invasive otitis externa [1]. This aggressive infection is usually caused by Pseudomonas aeruginosa, sometimes by fungal pathogens (mainly Aspergillus spp.), and in a minority of cases by other bacteria (such as Staphylococci, Proteus and Klebsiella) [2-4]. Besides an otologic origin of infection, sinusitis is the second major cause of skull base osteomyelitis. Although this is a rare complication,

\footnotetext{
* Correspondence: mihai.netea@radboudumc.nl

${ }^{\dagger}$ Equal contributors

Department of Internal Medicine and Radboudumc Center for Infectious

Diseases, Radboud University Medical Center, Geert Grooteplein Zuid 8, 6525 GA Nijmegen, The Netherlands
}

infections of the frontal, ethmoid, sphenoid and maxillary sinus can spread to the orbital and frontal bone, clivus and petrous apices. A distinct form of sinusitis is fungal rhinosinusitis. This has a broad clinical spectrum ranging from chronic forms with gradually progressing osteomyelitis to necrotizing angioinvasive disease. Aspergillus, Rhizopus and Fusarium are the most commonly identified fungi. Fungal skull base osteomyelitis due to Aspergillus is an infection with considerable morbidity and mortality rates up to $50 \%$. In addition to aggressive surgical debridement and systemic antifungal therapy, the mainstay of therapy includes, whenever possible, correction of the underlying immunologic defect.

Spores of Aspergillus are continuously inhaled and therefore fungal colonization of the upper airways is common. Despite this continuous exposure, invasive disease caused by Aspergillus in an immunocompetent host 
is very rare. Although some degree of immunosuppression may be present in patients with fungal SBO, often the only apparent risk factor identified is a chronic external otitis or an anatomical obstruction of the sinuses (e.g. nasal polyps or chronic inflammation of the mucosa). The extent of tissue invasion in these patients may vary depending on the underlying immune status of the host. In the present study we present six cases of skull base osteomyelitis due to Aspergillus fumigatus and Aspergillus flavus, in whom we investigated whether the innate and adaptive immune responses known to be important for antifungal host defense are defective.

\section{Methods}

\section{Volunteers and patients}

We describe six patients who were admitted to the Radboud University Medical Center with a culture-proven invasive Aspergillus osteomyelitis of the skull. All patients were diagnosed between September 2007 and July 2010. Charts were reviewed for data on demographics, risk factors, presenting symptoms, treatment, side effects, microbiology results, and clinical outcome. Response was defined according to the revised MSG/EORTC consensus group definition [5]. Patients and healthy volunteers, who served as healthy controls in the immunological experiments, were asked for blood donations. The blood samples were collected from patients and healthy volunteers after informed consent was obtained in accordance to Good Clinical practice, the Declaration of Helsinki, and the approval of the Arnhem-Nijmegen Ethics Committee (nr.2010/104).

\section{Stimuli}

E.coli lipopolysaccharide (LPS) $(10 \mathrm{ng} / \mathrm{ml})$ (TLR4 ligand, E. coli serotype O55:B5, Sigma-Aldrich St. Louis, MO USA); heat-killed Staphylococcus aureus clinical isolate (S. aureus) $\left(1 \times 10^{7} / \mathrm{ml}\right)$; heat-killed Candida albicans yeast ATCC MYA-3573 (UC820) (C. albicans) $\left(1 \times 10^{5} / \mathrm{ml}\right)$; heat-killed Aspergillus fumigatus clinical isolate V05-27 (A. fumigatus) conidia $\left(1 \times 10^{7} / \mathrm{ml}\right)$ were cultured and isolated as described previously [6].

\section{Peripheral blood mononuclear cells (PBMCs) isolation}

Fresh venous blood was drawn in $10 \mathrm{ml}$ EDTA tubes from patients and controls and processed in parallel continuously from PBMC isolation to cytokine measurement. Every patient gave blood only once. The blood was diluted 1:1 with Phosphate Buffered Saline (PBS). Subsequently PBMCs were isolated using Ficoll-paque (GE Healthcare, Zeist, The Netherlands) density gradient centrifugation. The PBMCs layer was collected and washed twice in cold PBS. Cells were reconstituted in RPMI-1640 culture medium (Dutch modification, Gibco, Invitrogen, Breda, The Netherlands) supplemented with $10 \mu \mathrm{g} / \mathrm{ml}$ gentamicin, $10 \mathrm{mM} \mathrm{L}$-glutamine and $10 \mathrm{mM}$ pyruvate (Gibco). The cells were counted with a particle counter (Beckmann Coulter, Woerden, The Netherlands) and the concentration was adjusted to $5 \times 10^{6} \mathrm{cells} / \mathrm{ml}$.

\section{PBMCs stimulation}

PBMCs were plated in a 96-well plate (Corning, NY, USA) at a final concentration of $2.5 \times 10^{6} / \mathrm{ml}$ in an end volume of $200 \mu \mathrm{l}$ per well. Either medium or stimuli were added. Cells were incubated at $37^{\circ} \mathrm{C}$ with $5 \% \mathrm{CO}_{2}$, after 24 or 48 hours or 7 days supernatants were collected and stored at $-20^{\circ} \mathrm{C}$. Seven-day stimulations were performed in the presence of $10 \%$ pooled human serum. All stimulations assays were performed in duplicates.

\section{Cytokines measurements}

Cytokines were measured in the cell culture supernatants using a commercial ELISA kit (IL-1 $\beta$, TNF $\alpha$, IL-17 and IL22: R\&D Systems; IL-6 and IFNy: Sanquin) according to the instructions supplied by the manufacturer. Proinflammatory cytokines production was measured after 24 hours, IFNY after 48 hours and the T helper cytokines IL-22 and IL-17 after 7 days of incubation.

\section{Statistical analysis}

The Mann-Whitney- $U$ test was used to detect differences between healthy controls and patients. A $p$-value of $<0.05$ was considered statistically significant $\left(^{*}=p<0.05, * *=p<\right.$ 0.01 and $* *=p<0.001)$. Graphs represent cumulative results of all performed experiments and are presented as mean \pm standard error of the mean. Data were analyzed with GraphPad Prism v 5.0.

\section{Results}

\section{Demographic characteristics of patients and controls} Patients

Three male and three female patients were included in the study with age ranging from 37 to 87 years (mean: 59.5 years). Infections were located in the sphenoid, mastoid or ethmoid bones with partial affection of sinus cavernosus, frontal or temporal lobe or orbita. All patients presented with cranial nerve palsy. In four cases the diagnosis was additionally confirmed by a positive histology. One patient developed Aspergillus osteomyelitis following trans-sphenoidal surgery for pituitary adenoma with a chronic myeloid leukemia (CML) in the past; two had a history of diabetes; all other patients had primary fungal infection of sinus or mastoid.

\section{Controls}

Three male and three female healthy volunteers were included in the control group; the age ranged from 24 to 60 years (mean: 42.7 years). Three of the patients were recruited from the blood bank via the blood donor service (Sanquin, Nijmegen, The Netherlands) and three 
were recruited directly to our department for blood donation. All volunteers were healthy and did not have an immunologically relevant medical history.

\section{Microbiology}

Culture and molecular identification confirmed the fungal infection and susceptibility to antifungal drugs was tested (Table 1). Galactomannan assay was performed in all patients on serum and in 4 patients on cerebrospinal fluid. All results were negative (index $<0.5$ ).

\section{Treatment, clinical outcome and blood sampling}

Surgical debulking was performed in all patients. All patients were initially treated with systemic antifungal drug therapy. Voriconazole was first line treatment in all patients. Four patients were treated with voriconazole monotherapy. One patient was concomitantly treated with liposomal amphotericine B during the first months of treatment. Posaconazole was used after induction treatment in two patients. Duration of therapy (from 4.5 to 35 months) was guided by clinical response and imaging, with follow-up from 8 to 38 months. Four patients had a complete response, one had a relapse, and one died due to respiratory failure. During infection the leukocytes count was normal in five of six patients, ranging from $6.6 \times 10^{9} / 1$ to $9.1 \times 10^{9} / 1$. One patient had a decreased leukocyte count of $3.1 \times 10^{9} / 1$ and slightly decreased numbers in the differential blood count. Three patients had normal and three patients had increased CRP values (11 mg/l, $14 \mathrm{mg} / \mathrm{l}$ and $32 \mathrm{mg} / \mathrm{l})$. Blood samples for immunological assays were taken after clinical improvement and response to the therapy: in three patients during the first month after onset of the antifungal therapy, while in three patients blood was collected after the pharmacological therapy had been finished and the infection did not recur.

\section{Patients with Aspergillus SBO do not differ from healthy} controls in their production of proinflammatory cytokines

To investigate the innate immune response, PBMCs isolated from six patients with Aspergillus SBO were stimulated with Aspergillus fumigatus, E. coli LPS and different pathogens and compared with healthy controls (Figure $1 \mathrm{~A}-\mathrm{C}$ ). Neither the unspecific LPS-stimulation, nor the pathogen-specific C. albicans, S. aureus, and disease-specific $A$. fumigatus stimulations showed differences in the production of the inflammatory cytokines IL-1 $\beta$, TNF $\alpha$ and IL-6.

\section{Patients with Aspergillus SBO are deficient in Aspergillus- induced IL-17 and IL-22 but not in IFNY production}

While the recognition and initiation of inflammatory cytokine responses revealed to be intact, we wanted to address the question whether defects in the acquired immune response might explain the high susceptibility to the fungal infection of the six fungal SBO patients included in this study. PBMCs were stimulated with $C$. albicans and $S$. aureus, serving as positive controls for the induction of IFN $\gamma$, IL-17 and IL-22, and A. fumigatus, to investigate pathogen-specific deficiencies. The Th1 response, shown by IFNY production, did not differ between patients with Aspergillus SBO and healthy controls (Figure 1D). In contrast, IL-22 and IL-17 production was significantly reduced after stimulation with both fungal pathogens C. albicans and A. fumigatus (Figure 1E-F).

\section{Discussion}

In this study, we describe the clinical presentation and immunological features of six patients with Aspergillus SBO. None of the patients were neutropenic at the time of the infection or had a known primary immunodeficiency. We hypothesized that specific defects in Aspergillus-specific innate and/or adaptive immune response would contribute to the unsuccessful fungal clearance and extent of the Aspergillus infection in our patients. Therefore, we investigated host responses in six patients with Aspergillus-SBO. While the innate responses were not different from a healthy control group, Th17 cytokines induced by fungal pathogens such as $C$. albicans and A. fumigatus were shown to be defective in the patients with Aspergillus SBO.

Table 1 Anti-fungal susceptibility of microbiological isolates

\begin{tabular}{llllllll}
\hline Case & Isolate & AMT $(\mathbf{m g} / \mathbf{L})$ & ITC $(\mathbf{m g} / \mathbf{L})$ & VOR $(\mathbf{m g} / \mathbf{L})$ & Anidula $(\mathbf{m g} / \mathbf{L})$ & POSA (mg/L) & CASPO (mg/L) \\
\hline $\mathbf{1}$ & A. fumigatus & $*$ & 1 & 1 & 0.063 & 0.25 \\
$\mathbf{2}$ & A. fumigatus & 1 & 1 & 2 & & $<0.016$ \\
$\mathbf{3}$ & A. fumigatus & 0.5 & 0.063 & 0.125 & 0.031 & 0.063 \\
$\mathbf{4}$ & A. fumigatus & 1 & 0.25 & 1 & & 0.031 \\
$\mathbf{5}$ & A. fumigatus & $* *$ & 1 & 0.063 & 1 & & 0.5 \\
$\mathbf{6}$ & A. flavus & 1 & & & &
\end{tabular}

*MIC impossible because of poor sporulation. Analysis of Cyp51A-gene: no TR/L98H.

**No isolate available for susceptibility testing.

MIC values of fungal isolates tested for Amphotericin B (AMT), Itraconazole (ITC), Voriconazole (VOR), Anidulafungin (Anidula), Posconazole (POSA) and Caspofungin (CASPO) are listed. 


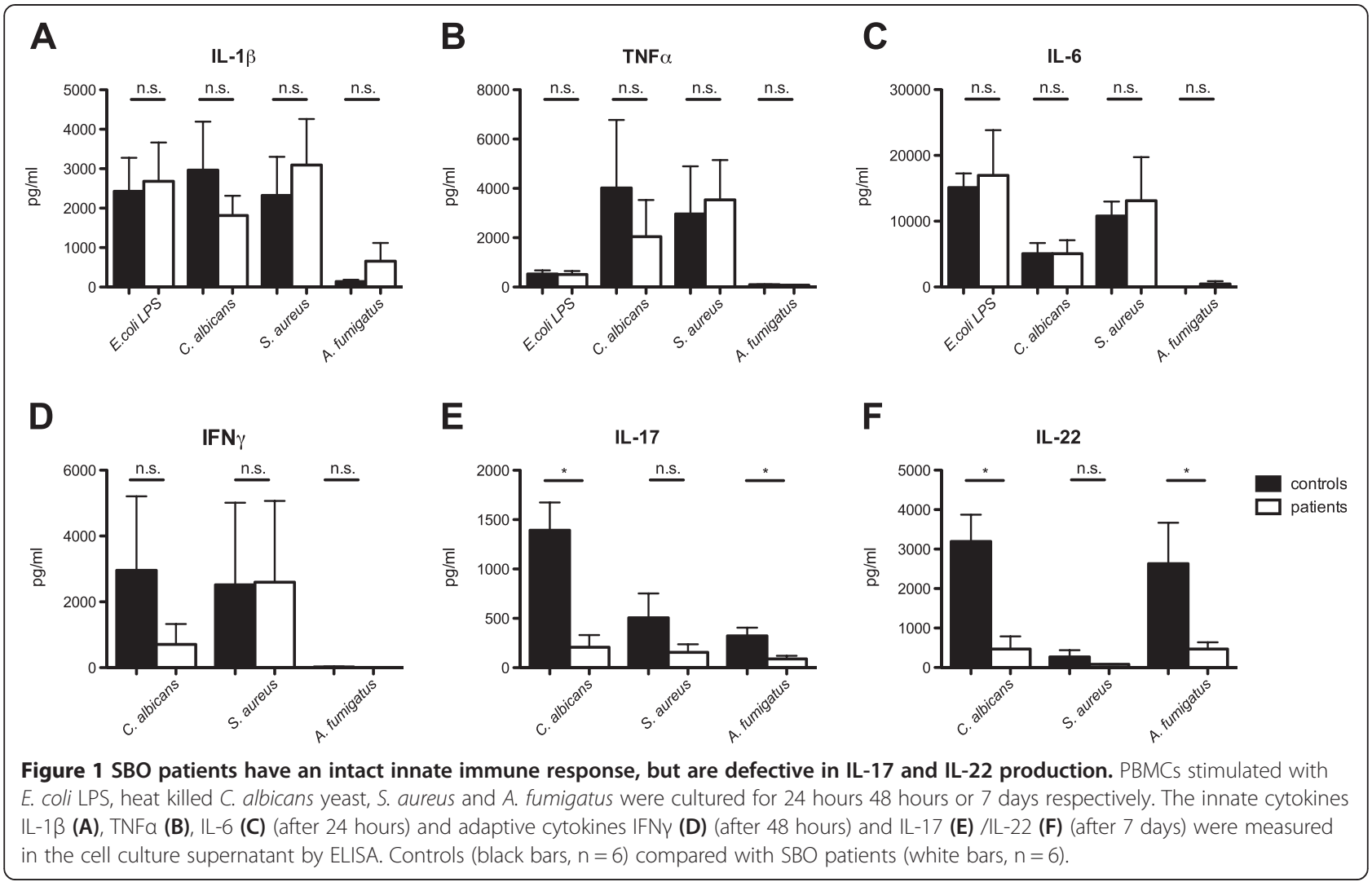

Aspergillus spp. can cause several forms of diseases dependent on the site of infection and immune status of the host. Aspergillus osteomyelitis is increasingly being reported [7] with approximately $15 \%$ of the cases affecting the skull base $[7,8]$. Aspergillus SBO is a severe complication of otitis externa or invasive sinonasal aspergillosis, in which most patients become infected via the tympanic cavity or the sinus [2].

The main risk factors described for invasive AspergillusSBO are systemic immunosuppression [9] and hematologic malignancies $(12 \%)[7,10]$. One patient included in this study suffered from chronic myeloid leukemia, and he was treated with the tyrosine kinase inhibitor imatinib at the time of the infection. Two patients had diabetes mellitus and three suffered from sinusitis, which have been reported in previous studies as risk factors $[7,10]$. Although almost all patients had normal leukocyte counts (one was slightly decreased), additional factors influencing the immune response such as the imatinib treatment, diabetes or the high age of one patient might affect the IL-17 response as well. Normal monocyte-derived cytokine levels point to a specific T-cell defect.

Early recognition and therapeutic intervention in invasive sinonasal aspergillosis with systemic antifungal therapy and surgical resection and/or debridement is important. In accordance with the current guidelines, all patients included in the present study were treated with surgical debridement of the infected bone and systemic antifungal drug therapy, of which voriconazole was the drug of first choice. We observed a mortality of 1 out of 6 in the patients included in the present study, which is in line with the reported poor clinical outcome of Aspergillus osteomyelitis, which has a 25\% 12-weeks mortality [11].

Why did our patients without an apparent severe immunodeficiency get invasive aspergillosis? Aspergillus spp. are an occasional commensal of the external ear and paranasal sinuses [12], but invasive disease is very rare. Chronic infection leading to damage of the epithelial barrier is an important entry for the fungus to infect the host. Recognition of Aspergillus will result in the production of proinflammatory cytokines that will recruit immune cells to clear the infection [13]. In the present study, patients with Aspergillus SBO showed normal production of the cytokines TNF $\alpha$, IL- $1 \beta$ and IL-6 after stimulation with $A$. fumigatus, $C$. albicans, the Gram-positive bacterium $S$. aureus, and the Gram-negative cell wall component E. coli LPS. Therefore, a defect in the production of monocyte-derived proinflammatory cytokines is unlikely to be the cause of the susceptibility of fungal SBO in our patients. Acquired adaptive T-helper responses also play an important role in anti-Aspergillus host defense. The protective role of IFN $\gamma$ in the Aspergillus-specific host response has been reported previously [14]. We did not observe a difference in Aspergillus-specific IFN $\gamma$ production; Candida-specific IFN $\gamma$ 
production showed a trend towards a lower IFNY production, but this was not significant. However, the Aspergillusspecific Th17 response was significantly lower in SBO patients compared to the healthy control group. In addition, this was also observed, when the cells were stimulated with Candida. IL-17 is a characteristic cytokine produced by Th17 cells. Th17 cells are crucial for neutrophil recruitment and controlling fungal invasion at the level of mucosae and skin [15]. Similar to IL-17, the IL-22 production was significantly decreased in SBO patients compared to healthy controls in the present study. IL-22 is also produced by Th17 cells [16] and shares many effector functions with IL-17 [17]. IL-22 plays a predominant role in mucosal host defense [18] by inducing anti-microbial peptides produced by epithelial cells, which can kill microorganisms directly [19].

In a previous study we have identified that IFNY treatment had beneficial effects on the immune status including IL-17 and IL-22 responses in a case series of patients with invasive fungal infections [20]. This suggests that adjuvant therapy with recombinant IFN $\gamma$ may improve the outcome of patients with a severe fungal SBO. Another treatment option would be to increase the differentiation into Th1 and Th17 cells by GM-CSF [21]. GM-CSF was shown to enhance the secretion of inflammatory cytokines [22] and antigen-presentation [23] under inflammatory conditions. It also induces the differentiation of progenitors cells into monocytes and granulocytes [24]. Therefore, GM-CSF might have beneficial effects on immune cells in the skin even in the setting of an IL-17/IL-22 deficiency [25]. One might speculate about supplementary therapy with recombinant IL-22, since IL-22 was shown to have beneficial effect in wound healing processes and pre-clinical studies have shown good toleration of administration of the drug [26].

We are aware of the fact that the sample size of six patients is inevitably small. A type I error of $5 \%$, meaning the likelihood to accept the hypothesis that Aspergillus SBO patients and control do not differ in their Aspergillus-specific IL-17 response was assumed. Using the calculated means, standard deviations of the experimental measurements and sample size of both groups we calculated a statistical power of $81 \%$. Thus, the Type II error, meaning the hypothesis that patients and controls differ in their IL-17 response is neglected, lay with $19 \%$ in an acceptable range for medical tests. Retrospectively, due to the differences observed, the sample size of 6 donors was the minimal size needed to detect statistically relevant differences. Further sample size independent calculation revealed an effect size of 1.5, which indicates that Aspergillus SBO has a statistically strong effect on the Aspergillus-specific IL-17 production.

Nevertheless, there are also some limitations of the study. One note of caution is that while patients and controls matched regarding their gender, the patients were in average older, although the 15 years difference is unlikely to explain the significant differences observed. Further, it remains unknown whether the defective IL-17 and IL-22 production was the consequence of a primary defect (e.g. genetic) or was secondary to a predisposing factor of the Aspergillus SBO patients (e.g. the antifungal treatment). Nevertheless, the present study contributes to understanding the specific defective host defense mechanisms underlying SBO due to Aspergillus.

\section{Conclusions}

This is the first study describing a deficiency in fungalinduced Th17 responses in patients with Aspergillus skull base osteomyelitis. Future studies are needed to validate this observation and its clinical implication, especially the potential beneficial effects of immunotherapy aimed to boost Th17 responses.

\section{Competing interests}

The authors declare that they have no competing interests.

\section{Authors' contributions}

CED, KLB, FL vd V, MGN designed and performed experiments; CED, FL vd V, MGN and KLB analyzed the experiments; CED, KLB, AS, FL vd $V$ and MGN wrote the manuscript; AS, B-J K and CPB-R critically revised the manuscript; CED, FL vd V, AS, B-J K, CPB-R, and MGN included patients. All authors read and approved the final manuscript.

\section{Acknowledgements}

F.vd V. was supported by a Veni grant of the Netherlands Organization for Scientific Research. M.G.N. was supported by a Vici grant of the Netherlands Organization for Scientific Research and an ERC Consolidator Grant (\#310372).

Received: 25 November 2014 Accepted: 12 March 2015

Published online: 21 March 2015

\section{References}

1. Chandler JR. Malignant external otitis and osteomyelitis of the base of the skull. Am J Otol. 1989;10(2):108-10.

2. Stodulski D, Kowalska B, Stankiewicz C. Otogenic skull base osteomyelitis caused by invasive fungal infection. Case report and literature review. Eur Arch Otorhinolaryngol. 2006;263(12):1070-6.

3. Rubin J, Yu VL. Malignant external otitis: insights into pathogenesis, clinical manifestations, diagnosis, and therapy. Am J Med. 1988;85(3):391-8.

4. Blyth CC, Gomes L, Sorrell TC, da Cruz M, Sud A, Chen SC. Skull-base osteomyelitis: fungal vs. bacterial infection. Clin Microbiol Infec t. 2011;17(2):306-11.

5. Segal BH, Herbrecht R, Stevens DA, Ostrosky-Zeichner L, Sobel J, Viscoli C, et al. Defining responses to therapy and study outcomes in clinical trials of invasive fungal diseases: Mycoses Study Group and European Organization for Research and Treatment of Cancer consensus criteria. Clin Infect Dis. 2008;47(5):674-83.

6. Netea MG, Warris A, Van der Meer JW, Fenton MJ, Verver-Janssen TJ, Jacobs LE, et al. Aspergillus fumigatus evades immune recognition during germination through loss of toll-like receptor-4-mediated signal transduction. J Infect Dis. 2003;188(2):320-6.

7. Gabrielli E, Fothergill AW, Brescini L, Sutton DA, Marchionni E, Orsetti E, et al. Osteomyelitis caused by Aspergillus species: a review of 310 reported cases. Clin Microbiol Infect. 2014;20(6):559-65.

8. Koehler P, Tacke D, Cornely OA. Aspergillosis of bones and joints - a review from 2002 until today. Mycoses. 2014;57(6):323-35.

9. Latge JP. Aspergillus fumigatus and aspergillosis. Clin Microbiol Rev. 1999;12 (2):310-50. 
10. Gupta S, Koirala J, Khardori R, Khardori N. Infections in diabetes mellitus and hyperglycemia. Infect Dis Clin North Am. 2007;21(3):617-38. vii.

11. Horn D, Sae-Tia S, Neofytos D. Aspergillus osteomyelitis: review of 12 cases identified by the Prospective Antifungal Therapy Alliance registry. Diagn Microbiol Infect Dis. 2009;63(4):384-7.

12. Vennewald I, Henker M, Klemm E, Seebacher C. Fungal colonization of the paranasal sinuses. Mycoses. 1999;42 Suppl 2:33-6.

13. Gresnigt MS, Netea MG, van de Veerdonk FL. Pattern recognition receptors and their role in invasive aspergillosis. Ann N Y Acad Sci. 2012;1273:60-7.

14. Cenci E, Perito S, Enssle KH, Mosci P, Latge JP, Romani L, et al. Th1 and Th2 cytokines in mice with invasive aspergillosis. Infect Immun. 1997;65(2):564-70.

15. van de Veerdonk FL, Plantinga TS, Hoischen A, Smeekens SP, Joosten LA, Gilissen C, et al. STAT1 mutations in autosomal dominant chronic mucocutaneous candidiasis. N Engl J Med. 2011;365(1):54-61.

16. Gresnigt MS, Becker KL, Smeekens SP, Jacobs CW, Joosten LA, van der Meer $J W$, et al. Aspergillus fumigatus-induced IL-22 is not restricted to a specific Th cell subset and is dependent on complement receptor 3. J Immunol. 2013;190(11):5629-39.

17. Liang SC, Tan XY, Luxenberg DP, Karim R, Dunussi-Joannopoulos K, Collins M, et al. Interleukin (IL)-22 and IL-17 are coexpressed by Th17 cells and cooperatively enhance expression of antimicrobial peptides. J Exp Med. 2006;203(10):2271-9.

18. Eyerich S, Eyerich K, Pennino D, Carbone T, Nasorri F, Pallotta S, et al. Th22 cells represent a distinct human $T$ cell subset involved in epidermal immunity and remodeling. J Clin Invest. 2009;119(12):3573-85.

19. Boniface K, Bernard FX, Garcia M, Gurney AL, Lecron JC, Morel F. IL-22 inhibits epidermal differentiation and induces proinflammatory gene expression and migration of human keratinocytes. J Immunol. 2005;174(6):3695-702.

20. Delsing CE, Gresnigt MS, Leentjens J, Preijers F, Frager FA, Kox M, et al. Interferon-gamma as adjunctive immunotherapy for invasive fungal infections: a case series. BMC Infect Dis. 2014;14:166.

21. King IL, Kroenke MA, Segal BM. GM-CSF-dependent, CD103+ dermal dendritic cells play a critical role in Th effector cell differentiation after subcutaneous immunization. J Exp Med. 2010;207(5):953-61.

22. Shortman K, Naik SH. Steady-state and inflammatory dendritic-cell development. Nat Rev Immunol. 2007;7(1):19-30.

23. Zhan Y, Carrington EM, van Nieuwenhuijze A, Bedoui S, Seah S, Xu Y, et al. GM-CSF increases cross-presentation and CD103 expression by mouse CD8 (+) spleen dendritic cells. Eur J Immunol. 2011;41(9):2585-95.

24. Waller EK. The role of sargramostim (rhGM-CSF) as immunotherapy. Oncologist. 2007;12 Suppl 2:22-6.

25. Meier K, Nanney LB. Emerging new drugs for wound repair. Expert Opin Emerg Drugs. 2006;11(1):23-37.

26. Muhl H, Scheiermann P, Bachmann M, Hardle L, Heinrichs A, Pfeilschifter J. IL-22 in tissue-protective therapy. Br J Pharmacol. 2013;169(4):761-71.

\section{Submit your next manuscript to BioMed Central and take full advantage of:}

- Convenient online submission

- Thorough peer review

- No space constraints or color figure charges

- Immediate publication on acceptance

- Inclusion in PubMed, CAS, Scopus and Google Scholar

- Research which is freely available for redistribution

Submit your manuscript at www.biomedcentral.com/submit 